\title{
Effects of an EEG Biofeedback Protocol on a Mixed Substance Abusing Population
}

\author{
William C. Scott, B.S.W., C.C.D.P., ${ }^{1}$ David Kaiser, Ph.D., ${ }^{2}$ \\ Siegfried Othmer, Ph.D., ${ }^{3}$ and Stephen I. Sideroff, Ph.D. ${ }^{4}$ \\ ${ }^{1}$ Neuropsychiatric Institute, University of California, Los Angeles, \\ California, USA and Restorative Technologies, \\ Santa Monica, California, USA \\ ${ }^{2}$ Department of Psychology, Rochester Institute of Technology, \\ Rochester, New York, USA \\ ${ }^{3}$ EEG Spectrum International Inc., Encino, California, USA \\ ${ }^{4}$ Department of Psychiatry and BioBehavioral Sciences, University of \\ California, Los Angeles, California, USA and Moonview Sanctuary, \\ Santa Monica, California, USA
}

\begin{abstract}
This study examined whether an EEG biofeedback protocol could improve outcome measures for a mixed substance abusing inpatient population. Method. One hundred twenty-one volunteers undergoing an inpatient substance abuse program were randomly assigned to the EEG biofeedback or control group. EEG biofeedback included training in Beta and SMR to address attentional variables, followed by an alpha-theta protocol. Subjects received a total of 40 to 50 biofeedback sessions. The control group received additional time in treatment equivalent to experimental procedure time. The Test of Variables of Attention (TOVA), and MMPI, were administered with both tester and subject blind as to group placement to obtain unbiased baseline data. Treatment retention and abstinence rates as well as psychometric and cognitive measures were compared. Results. Experimental subjects remained in treatment significantly longer than the control group
\end{abstract}


$(\mathrm{p}<0.005)$. Of the experimental subjects completing the protocol, $77 \%$ were abstinent at 12 months, compared to $44 \%$ for the controls. Experimental subjects demonstrated significant improvement on the TOVA $(\mathrm{p}<.005)$ after an average of 13 betaSMR sessions. Following alpha-theta training, significant differences were noted on 5 of the 10 MMPI- 2 scales at the $\mathrm{p}<.005$ level. Conclusions. This protocol enhanced treatment retention, variables of attention, and abstinence rates one year following treatment.

Keywords: EEG, biofeedback, EEG biofeedback, addiction treatment, chemical dependency, alpha-theta, TOVA, MMPI

\section{INTRODUCTION}

Alcohol and drug abuse is an ongoing societal and treatment problem $(1,2)$. While major resources have been employed to study and treat addiction, there has been little significant improvement in the success rate of treatment. Relapse rates remain high, typically over $70 \%$ (3-5). Gossop et al. (6) reported $60 \%$ of heroine addicts relapsed one year following addiction treatment.

Peniston and associates have demonstrated significantly higher abstinence rates with alcoholics when they incorporated EEG biofeedback into the treatment protocol (7-10). Eighty percent of subjects in these experiments were abstinent one-year posttreatment.

EEG biofeedback training is an operant conditioning technique used to reinforce or inhibit specific forms of EEG activity. In the alpha-theta protocol employed by the Peniston studies, low frequency EEG activity was reinforced. The alpha theta protocol was first demonstrated to be effective with posttraumatic stress disorder (11).

The efficacy of alpha-theta EEG biofeedback may lie in its ability to allow participants to better tolerate stress, anxiety, and anxiety-eliciting situations, which are particularly evident during the initial phases of recovery. This protocol was shown to significantly lower 13 of the scales of the Millon Clinical Multiaxial Inventory (MCMI), including anxiety, whereas traditional treatment produced decreases in only two of these scales (7). There have been, however, questions raised in the literature regarding the sample size, sample independence, and methodology in the Peniston et al. studies (12). Furthermore, there have been no controlled studies reported that extend these findings to other substances of abuse.

In addition to the psychological problems that substance abusers face in remaining abstinent, they also experience comorbid conditions that affect cognitive and attentional deficits. These deficits may be acquired from prolonged substance abuse (13-17), but the evidence also points to 
deficits that predate the abusing behavior (18). For example, in one study, approximately $35 \%$ of treatment-seeking cocaine abusers met the $D S M-I V$ criteria for childhood attention deficit hyperactivity disorder (19). Also, adult alcoholics report more residual-type attention deficit disorder (ADD) than controls (20). Low cognitive ability also has been shown to predict relapse after treatment at an alcohol treatment facility (21).

EEG biofeedback also has been used successfully to improve attentional, cognitive, and psychosocial functioning, including reductions in impulsivity (22-24). These and other studies have employed a protocol in which beta and SMR frequencies $(15-18 \mathrm{~Hz}$ and $13-15 \mathrm{~Hz}$, respectively) were operantly conditioned, while inhibiting theta frequencies, in remediating attentional and cognitive deficits in children and adults with ADD (25-28, 35). Given the relationship between cognitive/attentional impairment and addiction it would strengthen a treatment model to address these deficits.

In the present study, a beta/SMR EEG biofeedback training regimen was combined with an alpha-theta protocol in the treatment of a mixed substance abusing population. One expected objective was the enhanced ability of the subjects to focus on the treatment program, reduce impulsivity, and, thereby, increase program retention.

In order to extend the positive EEG biofeedback findings in the alcoholic population, an addict population was selected that included patients addicted to the following primary drugs: heroin, crack/cocaine, and methamphetamine, as well as alcohol.

\section{METHOD}

\section{Participants}

One hundred twenty-one volunteers from the Cri-Help, Inc. residential treatment program in the Los Angeles area participated in this study. There were 49 females and 72 males. They were 19 to 53 years of age, with a mean age of 32.4. The primary drug of choice reported at admission was $31 \%$ heroin, $28 \%$ crack cocaine, 26\% methamphetamine, $6 \%$ alcohol, and $9 \%$ other controlled substances; $94 \%$ were multiple-drug users.

Subjects determined to have a diagnosed psychotic or personality disorder (based on DSM-IV criteria), or a seizure disorder, were excluded. Subjects were randomly assigned to the EEG biofeedback plus conventional treatment group (60 experimental subjects) or the conventional treatment-only group (61 control subjects). 
Subjects were provided informed consent before participating in this experiment, approved by the UCLA Human Subjects Protection Committee.

\section{Procedures}

All subjects received treatment based on the Minnesota Model 12-step oriented program described by Stinchfield and Owen (29) supported by group, family, and individual counseling. In addition, the experimental group received 40-50 EEG biofeedback sessions. The control group received additional treatment time equivalent to the biofeedback sessions.

Experimental subjects underwent two sessions of EEG biofeedback training (45 minutes per session) five days a week for four to five weeks. EEG biofeedback was performed on a Neurocybernetics 2- Channel EEG biofeedback system.

In Phase I, experimental subjects underwent 10-20 sessions of BetaSMR EEG biofeedback in which operant conditioning was used to augment either $15-18 \mathrm{~Hz}$ (beta) or $12-15 \mathrm{~Hz}$ (SMR) EEG activity. At the same time, training attenuated elevated activity in the $2-7 \mathrm{~Hz}$ (theta) and $22-30 \mathrm{~Hz}$ (high beta) ranges. Active bipolar electrode placement was at C3-FPZ for beta and at C4-PZ for SMR, based on the international 10-20 system of electrode placement (30).

The starting protocol consisted of beta training $50 \%$ of the time and SMR training $50 \%$ of the time. These percentages would be altered based on changing symptomatology and TOVA results (31) with inattentive or impulsive profiles resulting in increased beta or SMR training, respectively.

After 10 Beta-SMR EEG biofeedback sessions, participants were reassessed with the TOVA. If a participant scored within the normal range (i.e., scores of 85 or above), he or she began alpha-theta training. If the TOVA remained abnormal after the initial 10 Beta-SMR sessions, 5 or 10 additional Phase 1 treatments were administered. It took a median of 10 BetaSMR sessions with a mean of 13 sessions for the TOVA to normalize for the experimental subjects.

In Phase II, subjects underwent 30 sessions of alpha-theta training. The frequency range for alpha was $8-11 \mathrm{~Hz}$ and for theta it was $5-8 \mathrm{~Hz}$. The initial sessions were used to train down alpha levels that were above $12 \mu \mathrm{V}$ (peak to peak), while augmenting theta, until there was "crossover." This was defined as the point at which the alpha amplitude drops below the level of theta. Subsequent to the first achievement of crossover, both alpha and theta frequencies were augmented.

Before initial crossover was achieved, excess EEG activity in the range of $15-30 \mathrm{~Hz}$ was inhibited. This was intended to reduce muscle tension and to quiet the mind. After crossover was achieved, the $2-5 \mathrm{~Hz}$ frequency range 
also was inhibited. This was intended to discourage the sleep transition during low-arousal states.

Each alpha-theta session began with the subject sitting in a chair with eyes closed. The active electrode was placed at $\mathrm{Pz}$ with a left-ear reference (A1). The right earlobe was connected to circuit ground. Two distinct tones were employed for alpha and theta reinforcement, with the higher pitched sound used to index the higher-frequency alpha band.

At the start of each session, the technician spent 3-5 minutes reading a script of guided imagery to the experimental subject that dealt with identified essential elements of maintaining abstinence. These included ongoing regular attendance at 12-step meetings; weekly meetings with a sponsor, expanding the individuals identified comfort zones, and mental exercises dealing with cue extinction and relapse rejection.

After the guided imagery, it was made clear to the subject that the objective of the training did not involve explicit rehearsal of the script during the EEG biofeedback. Subjects reporting previous meditative practices were asked not to use them during the training, since meditation has been observed to override alpha-theta reinforcement effects. Following the alpha-theta training, clients were given the opportunity to process their experience.

When it appeared that sleep might be occurring during training, subjects were told prior to their next session to move a limb if they heard the technician say either, "Right foot, left foot, right hand, or left hand." At points where the subject's delta activity $(2-5 \mathrm{~Hz}$ EEG) started to elevate, as well as at their highest amplitudes (indications of sleep onset), the limb commands were given to determine responsiveness. The delta amplitude value at which the subject transitioned to nonresponsiveness was documented. Subsequently, during sessions where delta was elevating toward nonresponsiveness levels, the feedback sounds were inhibited in order to discourage the sleep transition.

\section{Measurements}

Tests were administered prior to training, after Beta-SMR training (Phase 1) and after alpha-theta training (Phase 2) for experimental subjects and at commensurate points in time for the control group (typically 1, 16, and 46 days into the research program). All subjects had acclimated to the institutional setting for a minimum of 7 days prior to testing. The initial testing was accomplished with subjects and experimenters blind to group placement.

The TOVA was administered to assess attentional and cognitive functions (31-33). The Minnesota Multiphasic Personality Inventory (MMPI-2) was administered at the start of the study and again at 46 days. 
Patient abstinence was determined by collateral contacts in addition to self-report. Follow-up interviews for this purpose took place at 3-month intervals over a 12-month period. Research subjects gave permission to contact individuals who were intimately involved in their recovery. These individuals were their 12-step sponsors, family members, and those people referring the subject into the program. Subjects who used substances beyond one 4-week window were considered to have fully relapsed. Those whose relapse duration was within a single 4 -week window were categorized as a brief relapse (Please see appendix for procedures flow chart.).

\section{RESULTS}

\section{Days in Treatment}

Length of stay in treatment averaged 138 days for experimental subjects and 101 days for controls. This difference was significant $t(119)=-3.07$, $p<0.005$. Median length of stay was 147 days for experimental and 103 days for control subjects. Figure 1 shows retention in the program over the first 12 weeks of the program. As can be seen, at the end of this period, $46 \%$ of control subjects had dropped out of treatment, compared to only $24 \%$ of those who received EEG biofeedback. A chi-square analysis demonstrated a significant difference in drop-out rate between experimental and control groups over the 12 -week period $\left[X^{2}(n=121)=6.29, p<.05\right.$. $]$ There was no

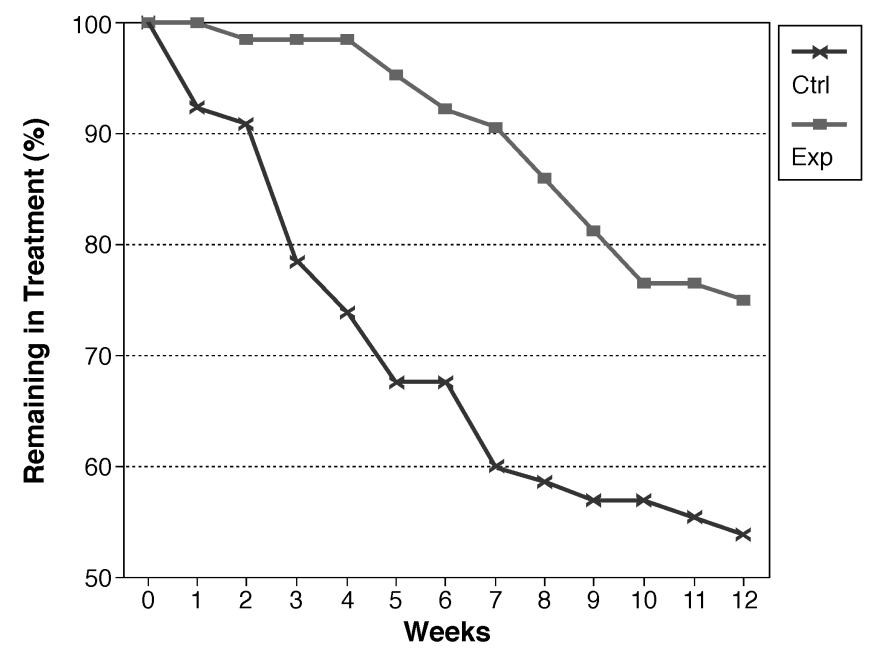

Figure 1. Effect of the EEG biofeedback protocol on patient retention for control $(n=61)$ and experimental $(n=60)$ subjects. 
significant interaction between drug type used (stimulant vs. sedating drugs) and days remaining in treatment $[\mathrm{F}(1,118)=.004, \mathrm{~ns}]$.

\section{Abstinence Rate}

Figure 2 presents the data for the 103 subjects who had reached their 12 month poststudy status. This includes 55 experimental and 48 control subjects. Of these subjects, there were 7 experimental and 17 control subjects who dropped out of treatment prior to completing the study (the initial 45 days), while there were 4 control subjects and 1 experimental subject who could not be contacted at the 12-month interval.

Of the remaining experimental subjects who completed the study and were assessed at 12 months, 36 of $47(77 \%)$ were abstinent. This included 8 subjects who had one brief relapse period of less than 30 days during the year. Of the control subjects who completed the study, there were 12 of 27 subjects (44\%) who were abstinent. This included 1 subject who had one brief relapse period of less than 30 days. A chi-square analysis demonstrated a significant difference between one year abstinence rates of the experimental group versus the control group $\left(X^{2} 2(74)=7.78 \mathrm{p}<0.01\right)$. There was no significant interaction between drug type used (stimulant versus depressant) and abstinence rate $[\mathrm{F}(1,113)=.844, \mathrm{p}>.05]$.

\section{MMPI-2 Data}

Figure 3 presents pre and posttraining MMPI data, including the 10 clinical scales and 3 validity scales, for the experimental and control groups. Subjects with Lie scores greater than 70 on either pre or posttraining tests were excluded from analysis $(n=3,2$ experimental and 1 control). A univariate

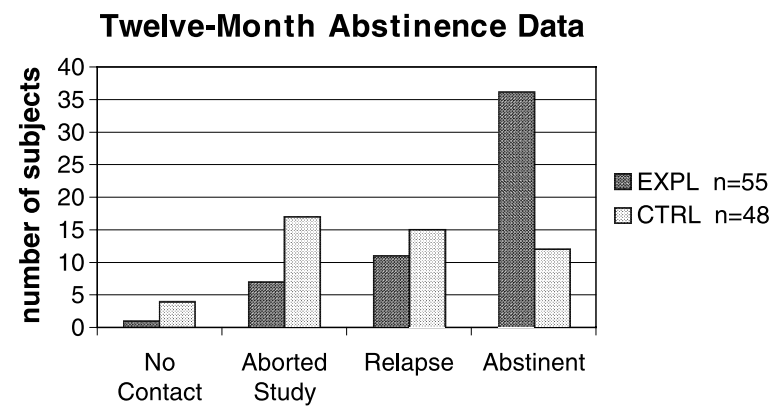

Figure 2. Twelve-month follow-up abstinence data for experimental $(\mathrm{n}=55)$ and control $(n=48)$ groups. 

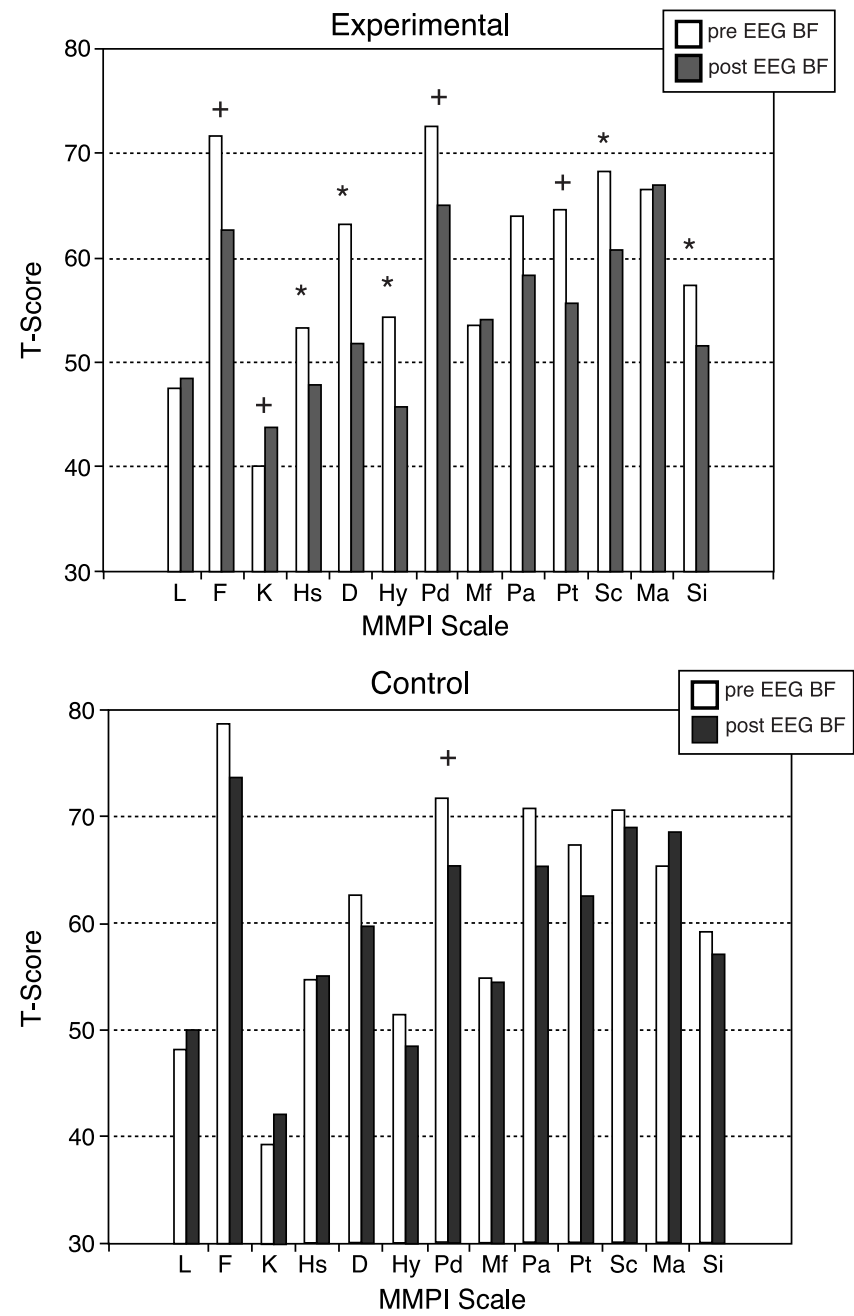

Figure 3. Change in 10 MMPI clinical scales and 3 validity scales for the experimental group $(n=50)$ and the controls $(n=33)(+p<.05, * p<.005)$.

mixed-design analysis of variance (ANOVA) was used to evaluate the effects of the experimental protocol compared to controls on the 10 clinical scales.

As shown in Figure 3, the experimental group's changes exhibited significant improvement compared with the changes in the control subjects ( $\mathrm{p}<0.005$ ), on the Hs (Hypochondriasis), $\mathrm{F}(1,81)=14.087$; D (Depression), $\mathrm{F}(1,81)=48.129$; Hy (Conversion Hysteria), $\mathrm{F}(1,81)=32.682$; Sc (Schizophrenia), $\mathrm{F}(1,81)=15.241$; and $\mathrm{Si}$ (Social Introversion) scales, $\mathrm{F}(1,81)=$ 24.647, $\mathrm{p}<.005$. The experimental group also improved on the $\mathrm{Pt}$ 


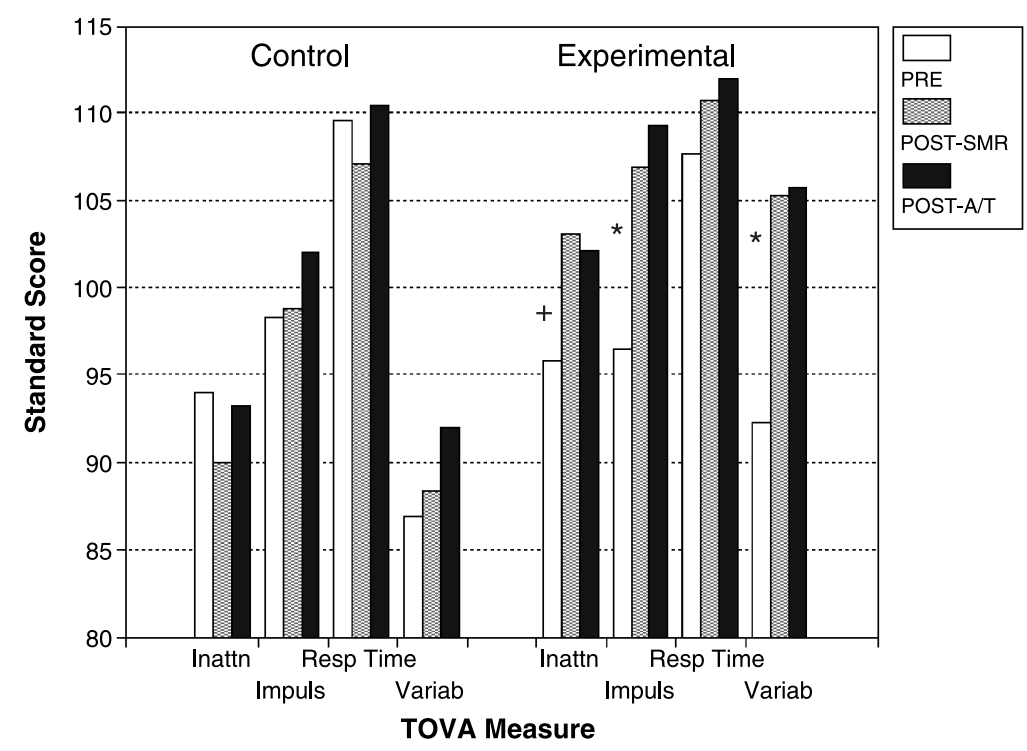

Figure 4. TOVA standard scores for experimental and control groups for pretraining, post-SMR, and post-alpha-theta assessments $(+\mathrm{p}<.05, * \mathrm{p}<.005)$.

(Psychasthenia) scale, although the difference between groups on this scale was not significant $F(1,81)=1.727, p>.05$. Both groups improved on the Pd (Psychopathic Deviate) scale, F(1, 81) $=29.016 ; \mathrm{F}(1,81)=12.832, \mathrm{p}<$ .05 , respectively.

\section{TOVA}

Mean TOVA standard scores are presented for both groups in Figure 4 (42 experimental, 28 controls). More participants were tested but only those who provided scores from all three test periods (baseline, post-SMR, post-alphatheta) were analyzed. There was no significant difference between groups in initial baseline TOVA scores $[\mathrm{F}(1,303)=1.333, \mathrm{p}>.05]$. A univariate, mixeddesign ANOVA was used to compare the two groups on four dependent measures of the TOVA: inattention (percent omission), impulsivity (percent commission), response time, and response variability. Low scores were truncated at four standard deviations below normal.

As can be seen in Figure 4, the experimental group exhibited significant improvement in impulsivity and variability measures in response to BetaSMR training $\mathrm{F}(1,68)=18.749 ; \mathrm{p}<.005$ whereas no comparable change was found for the control group $F(1,68)=19.405 ; p>0.05$. Experimental subjects also demonstrated significant improvement in inattention; however, the score 
only marginally differed from that of the control group $F(1,68)=5.549$ $(\mathrm{p}<.05)$. TOVA scores were not further enhanced by either the alpha-theta training nor 30 additional days of treatment.

\section{DISCUSSION}

The results of this study support the efficacy of EEG biofeedback training in an inpatient drug treatment program. Success was determined by length of time in treatment, or treatment retention, as well as by abstinence rates one year after termination of treatment. Results were further supported by positive changes in attentional variables, and positive changes on the MMPI 2. These findings extend the previous research findings employing alpha-theta EEG biofeedback with an alcoholic population, to other substances of abuse.

The present study employed a Beta-SMR protocol prior to the alpha-theta procedure previously used in addiction studies (7-10). Beta-SMR training previously had been shown to be effective in remediating attentional and cognitive deficits. Results of baseline performance testing using the TOVA did not demonstrate that this population had significantly below average attentional indices. However, testing following the Beta-SMR protocol showed that this procedure improved these test measures for the experimental subjects, particularly impulsivity and variability. This result may partly account for the improved treatment retention of this group.

It has been shown that time in treatment is one of the best predictors of remaining abstinent (34). In the present study, the experimental subjects averaged 136 days in treatment. This compared to 98 days for the control population. In addition, treating therapists reported that they noticed experimental subjects appearing more cooperative and more attentive as EEG biofeedback progressed. This subjective observation should be a focus in future studies with a more systematic observation of subjects' behavior.

There were 8 experimental subjects who used briefly (less than 30 days) but were abstinent at the 12-month follow-up, and there was 1 subject from the control group who had this experience. It has been noted in the previous alpha-theta treatment studies that patients report dysphoria when they used a substance following the EEG biofeedback protocol (8). Some of the experimental subjects in this study had similar experiences. This may indicate that a more fundamental neurophysiological change had taken place as a result of the treatment. Peniston and Kulkosky (7) for example, noted that experimental subjects receiving EEG biofeedback did not show increased circulating beta-endorphin levels, an index of stress, which was found in the control group.

It can be noted that once the EEG biofeedback was concluded, at week five, the subsequent attrition rates became indistinguishable between the two 
groups. It may be useful in future studies to extend the length of the biofeedback training to see if it has further impact on experimental results.

One of the more striking findings of the present study and similar to the Peniston (8) results, is the positive change noted in the MMPI. The experimental subjects showed significant improvement in five of the clinical scales: Hypochondriasis, Depression, Hysteria, Schizophrenia, and Social Introversion. These changes indicate a lowered level of general distress or discomfort. More specifically there may be a reduced sense of alienation and depression, as well as defensiveness. These are vital factors in recovery.

The present study did not demonstrate differential effectiveness of the EEG biofeedback protocol for sedative or stimulant drug abusers. This should be a focus of future research in which larger numbers of subjects are employed. Both groups of subjects appeared to benefit from this protocol. If the lack of dependency on drug type is confirmed, the case can be made that alpha/theta training addresses core issues in addiction rather than drugspecific aspects of dependency.

In the present study, one-year abstinence was determined by collateral contacts in addition to self-report. These individuals were reliable sources who were intimately connected to the recovery process, including their 12step sponsors, family members, and those people referring the subject into the program. Future research results should be supported by incorporating urine testing as a further corroboration of abstinence.

Since EEG-based reinforcement was such a prominent constituent of the experimental program, the question arises as to whether the benefits of training could also be documented through observable EEG changes. The present study was not designed to analyze the appropriate artifact free data. Future research should incorporate methodology to record and analyze the appropriate quantitative EEG data.

It is important to place the results of this study in the context of the longstanding difficulty in achieving successful abstinence with the drug-abusing population. EEG biofeedback appears to promote and support positive change in the level of neurophysiological and psychosocial functioning in the addict, as well as enhancing treatment retention. It therefore constitutes a promising approach that now requires additional study for further validation as well as to elucidate operative mechanisms to optimize the procedures, and to facilitate integration into standard treatment programs.

\section{CONCLUSION}

The present study supports the efficacy of an EEG biofeedback protocol as adjunctive therapy in an in-patient drug treatment program. This protocol appears to be beneficial for both sedative as well as stimulant substances of 
abuse. Success was determined by length of time in treatment as well as by abstinence one year after termination of treatment. Supportive data were provided through attentional/cognitive and psychological assessments. These findings extend the research employing alpha-theta EEG biofeedback with an alcoholic population to other drugs of abuse.

\section{APPENDIX}

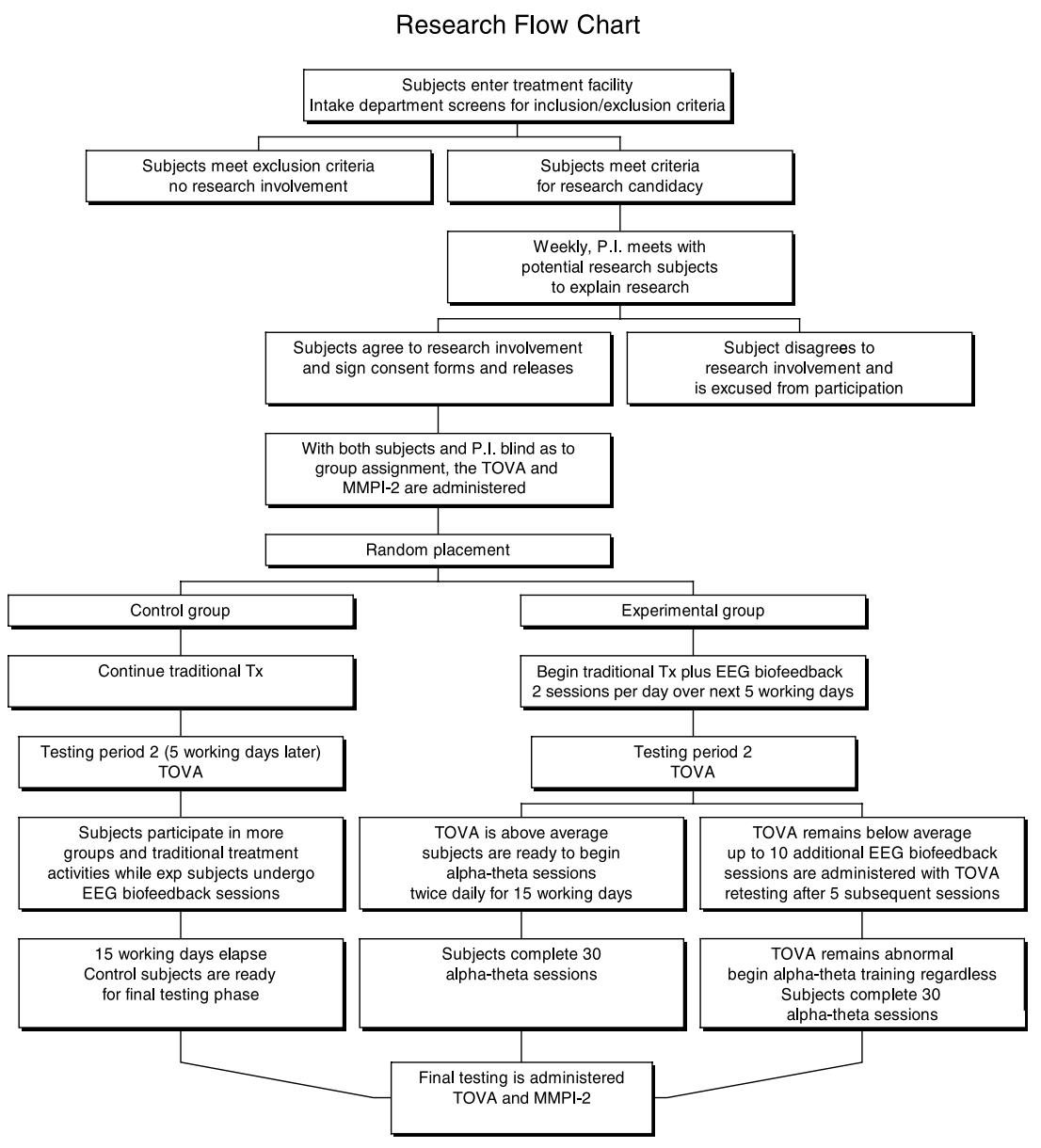

\section{ACKNOWLEDGMENTS}

We wish to thank Marcus Sola (CRI-Help, Chairman of the Board), Jack Bernstein (CRI-Help, CEO), and Marlene Nadel (CRI-Help, Clinical 
Supervisor) for their participation and willingness to add an innovative approach to their existing treatment model. Thanks also to the CRI-Help Board of Directors for providing funding for this project. We also thank EEG Spectrum International for their donation of a Neurocybernetics EEG biofeedback system. We wish to thank Don Theodore, MA, MFT and Leslie Ruddock, BA (Research Technicians) who administered all EEG biofeedback protocols and coordinated subject sessions with the traditional treatment team. We also thank Susan Othmer who shared her knowledge of beta/SMR protocols and for case consultation. Thanks also to Meredith Sagan for her consultation and critical review of the manuscript. National Computer Systems (NCS Assessments) donated administrations of a self-scoring computerized version of the MMPI-2. Universal Attention Disorders, Inc. contributed administrations of the Test of Variables of Attention (TOVA).

\section{REFERENCES}

1. Fagan J. Women and drugs revisited: female participation in the cocaine economy. Special Issue: drugs and crime revisited. J Drug Issues 1994; 24:179-225.

2. Yu J, Williford WR. Alcohol, other drugs, and criminality: a structural analysis. Am J Drug Alcohol Abuse 1994; 20:373-393.

3. Higgins ST, Budney AJ, Bickel WK, Badger G, Foerg F, Ogden D. Outpatient behavioral treatment for cocaine dependence: one year outcome. Exp Clin Psychopharmacol 1995; 3:205-212.

4. Kang S, Kleinman PH, Woody GE, Millman RB, Todd TC, Kemp J, Lipton DS. Outcomes for cocaine abusers after once-a-week psychosocial therapy. Am J Psychiatr 1991; 148:630-635.

5. Marlatt GA, Gordon JR. Determinants of relapse: implications for the maintenance of behavior change. In: Davidson P, ed. Behavioral Medicine: Changing Health Lifestyles. New York: Brunner/Mazel, 1979:410-452.

6. Gossop M, Stewart D, Browne N, Marsden J. Factors associated with abstinence, lapse or relapse to heroin use after residential treatment: protective effect of coping responses. Addiction 2002; 97(10):1259-1267.

7. Peniston EG, Kulkosky PJ. Alpha theta brainwave training and betaendorphin levels in alcoholics. Alcoholism: Clin Exp Res 1989; 13(2):271-279.

8. Peniston EG, Kulkosky PJ. Alcoholic personality and alpha-theta brainwave training. Med Psychother: Int J 1990; 3:37-55.

9. Peniston EG, Marrinan DA, Deming WA, Kulkosky PJ. EEG alphatheta brainwave synchronization in Vietnam theater veterans with combat-related post-traumatic stress disorder and alcohol abuse. Adv Med Psychother 1993; 6:37-50. 
10. Saxby E, Peniston EG. Alpha-theta brainwave EEG biofeedback training: an effective treatment for male and female alcoholics with depressive symptoms. J Clin Psychol 1995; 51:685-693.

11. Peniston EG, Kulkosky PJ. Alpha-theta brainwave neurofeedback therapy for Vietnam veterans with combat-related posttraumatic stress disorder. Med Psychother: Int J 1991; 4:47-60.

12. Graap K, Freides D. Regarding the database for the Peniston alpha-theta EEG biofeedback protocol. Appl Psychophysiol Biofeedback 1998; 23(4):265-275.

13. Ardila A, Rosselli M, Strumwasser S. Neuropsychological deficits in chronic cocaine abusers. Int J Neurosci 1991; 5:73-79.

14. Arria AM, Tarter RE, VanThiel DH. Liver-brain relations in alcoholics. Alcohol Health Res World 1990; 14:112-117.

15. Hambidge DM. Intellectual impairment in male alcoholics. Alcohol Alcohol 1990; 25:555-559.

16. Marchesi C, de Risio C, Campanini G, Maggini C. TRH test in alcoholics: relationship of the endocrine results with neuroradiological and neuropsychological findings. Alcohol Alcohol 1992; 27:531537.

17. O'Mahony JF, Doherty B. Intellectual impairment among recently abstinent alcohol abusers. Br J Clin Psychol 1996; 35:77-83.

18. Pogge DL, Stokes J, Harvey PD. Psychometric vs. attentional correlates of early onset alcohol and substance abuse. J Abnorm Child Psychol 1992; 20:151-162.

19. Carroll KM, Rounsaville BJ. History and significance and childhood attention deficit disorder in treatment-seeking cocaine abusers. Compr Psychiatry 1993; 34:75-82.

20. Nixon SJ, Tivis R, Parsons OA. Behavioral dysfunction and cognitive efficiency in male and female alcoholics. Alcohol: Clin Exp Res 1995; 19:577-581.

21. Miller L. Predicting relapse and recovery in alcoholism and addiction: neuropsychology, personality, and cognitive style. J Cocaine Abuse Treat 1991; 8:277-291.

22. Lubar JF, Swartwood MO, Swartwood JN, O'Donnell PH. Evaluation of the effectiveness of EEG biofeedback training for ADHD in a clinical setting as measured by changes in T.O.V.A. scores, behavioral ratings, and WISC-R performance. Biofeedback Self-Regul 1995; 20:83-99.

23. Lantz D, Sterman MB. Neuropsychological assessment of subjects with uncontrolled epilepsy: effects of EEG feedback training. Epilepsia 1988; 29(2):163-171.

24. Othmer S, Othmer SF, Kaiser DA. EEG Biofeedback: training for AD/ $\mathrm{HD}$ and related disruptive behavior disorders. In: Incorvaia JA, MarkGoldstein BS, Tessmer D, eds. Understanding, Diagnosing, and 
Treating AD/HD in Children and Adolescents, an Integrative Approach. New York: Aronson Press, 1999:235-318.

25. Cartozzo HA, Jacobs D, Gevirtz RN. EEG Biofeedback and the remediation of ADHD symptomatology: a controlled treatment outcome study. Presented at the Annual Conference of the Association for Applied Psychophysiology and Biofeedback, Cincinnati, Ohio, March 1995.

26. Linden M, Habib T, Radojevic V. A controlled study of the effects of EEG biofeedback on cognition and behavior of children with attention deficit disorders and learning disabilities. Biofeedback Self-Regul 1996; 21:35-50.

27. Rossiter TR, La Vaque TJ. A comparison of EEG biofeedback and psychostimulants in treating attention deficit/hyperactivity disorder. J Neurother 1995; 1:48-59.

28. Thompson L, Thompson M. Neurofeedback combined with training in metacognitive strategies: effectiveness in students with ADD. Appl Psychophysiol Biofeedback 1998; 23(4):243-263.

29. Stinchfield R, Owen P. Hazelden's model of treatment and its outcome. Addict Behav 1998; 23(5):669-683.

30. Jasper HH. The 10-20 system of the international federation. Electroencephalogr Clin Neurophysiol 1958; 10:371-375.

31. Dupuy TR, Greenberg LM. TOVA Manual. Los Alamitos, CA: Universal Attention Disorders, 1989.

32. Greenberg LM, Waldman ID. Developmental normative data on the Test of Variables of Attention (T.O.V.A.). J Child Psychol Psychiatry 1993; 34:1019-1030.

33. Kane RL, Kay GG. Computerized assessment in neuropsychology: a review of tests and test batteries. Neuropsychol Rev 1992; 3:1-117.

34. Ciraulo DA, Piechniczek-Buczek J, Iscan EN. Outcome predictors in substance use disorders. Psychiatr Clin North Am 2003; 26(2):381409.

35. Egner T, Gruzelier JH. Learned self-regulation of EEG frequency components affects attention and event-related brain potentials in humans. Neuroreport 2001; 12:4155-4159. 